\title{
Los nuevos patrones de crecimiento de la OMS
}

\section{Comparación con otras gráficas de crecimiento}

\author{
Ekhard E. Ziegler Steven E. Nelson \\ Departamento de Pediatría, Universidad de lowa, lowa City, lowa, EE.UU.
}

\section{Palabras clave}

Patrones de crecimiento de la OMS · Gráficas de crecimiento

\section{Extracto}

Los nuevos patrones de crecimiento de la Organización Mundial de la Salud (OMS) fueron creados con la intención de generar patrones de crecimiento globalmente aplicables que describiesen el crecimiento de los niños, tal como acontece en condiciones nutricionales óptimas y en ausencia de impedimentos externos. En otras palabras, representan el crecimiento infantil tal como debe ser. Los datos para las nuevas gráficas procedían de niños de 6 países, que vivían en circunstancias favorables. Además, se excluyeron los datos de algunos sujetos situados en los extremos del peso para talla. De forma nada inesperada, los nuevos patrones de crecimiento de la OMS diferían de otras gráficas de crecimiento en varios aspectos. Debido a las numerosas diferencias, sólo pueden efectuarse unas pocas aseveraciones generales: (1) Durante los 6 primeros meses de vida, el peso y la longitud de la OMS en todos los percentiles son mayores que el peso y la longitud de cualquier otra gráfica. (2) Durante los segundos 6 meses de vida, y sucesivamente hasta el segundo año de vida, el peso de la OMS (aunque no la longitud) es menor que el peso reflejado en otras gráficas. (3) Entre 2 y 5 años de vida, el peso de la OMS tiende a situarse en el extremo inferior del espectro, especialmente en los percentiles más bajos, mientras que Euro-Growth ocupa el extremo superior para el peso en todos los percentiles. (4) La evaluación funcional muestra que las gráficas de la OMS identifican menos niños de 1 a 2 años de edad con peso inferior al ideal y más niños de 2 a 5 años con sobrepeso, en comparación con otras gráficas.

Copyright $\odot 2008$ Nestec Ltd., Vevey/S. Karger AG, Basel
Las gráficas de crecimiento son herramientas importantes, tanto para la evaluación del crecimiento y el estado nutricional de niños individuales (uso clínico), como para la evaluación de grupos de niños (uso epidemiológico). Las gráficas de crecimiento son percibidas como representativas del crecimiento normal de los niños y, en consecuencia, son portadoras de autoridad normativa. Aunque numerosos países poseen gráficas de crecimiento nacionales, éste no es el caso en una inmensa mayoría de ellos. Para el uso en tales países, así como para facilitar comparaciones internacionales, existe la necesidad de disponer de gráficas de crecimiento universales. Para satisfacer esta necesidad, la Organización Mundial de la Salud (OMS) adoptó en 1978 gráficas de crecimiento que habían sido confeccionadas por el Centro Nacional de Estadística Sanitaria (NCHS) de los Estados Unidos [1]. Las gráficas llegaron a conocerse con la denominación de gráficas de crecimiento NCHS/ OMS.

Sin embargo, pronto llegó a hacerse evidente que las gráficas NCHS/OMS no representaban muy fielmente el crecimiento de los lactantes. En comparación con el peso de lactantes alimentados con leche materna en Europa y Estados Unidos [2], el peso para la edad de las gráficas NCHS/OMS era, según se comprobó, indebidamente bajo en los 6 primeros meses de vida, mientras que en los segundos 6 meses el peso del NCHS tendía a ser mayor que el de los lactantes alimentados con leche materna [3]. Se creyó que estas imperfecciones derivaban principalmente de dos características del estudio del crecimiento subyacente. En primer lugar, los datos antropométricos durante el primer año de vida eran demasiado espaciados (cada 3 meses). Segundo, pocos de los lactantes fueron alimentados enteramente con leche mater-

\section{KARGER \\ Fax +4161306 1234 E-Mail karger@karger.ch} www.karger.com
(C) 2008 Nestec Ltd., Vevey/S. Karger AG, Basel 0252-8185/07/0653-0111\$23.50/0

Accesible online en: www.karger.com/ans
Ekhard E. Ziegler, MD

Department of Pediatrics

University of Iowa, A 136 MTF, 2501 Crosspark Rd

Iowa City, IA 52242 (USA)

Tel. +1 319335 4570, Fax +1 319335 4856, E-Mail ekhard-ziegler@uiowa.edu 
na, y de los que sí, muchos fueron amamantados durante sólo un corto periodo de tiempo. También se consideró un inconveniente que los sujetos vivieran en una única zona geográfica de Estados Unidos. Un Comité de Expertos convocado por la OMS determinó que las gráficas NCHS/OMS eran inapropiadas e insuficientes y recomendó la creación de nuevas gráficas para uso global [3]. Se inició un estudio multicéntrico de referencia sobre crecimiento y entre 1997 y 2003 se recogieron datos para nuevas gráficas representativas internacionalmente de niños entre el nacimiento y los 5 años. Los nuevos Patrones de Crecimiento de la OMS para uso global se publicaron en 2006 [4].

Tradicionalmente, las gráficas de crecimiento describían el crecimiento de niños considerados normales y sanos y que habitaban en una zona geográfica determinada o bien en un país individual, como en el caso de las gráficas NCHS [1], o en un grupo de países, como en el caso de las gráficas Euro-Growth [5]. Dichas gráficas reciben el nombre de referencias del crecimiento. Las nuevas gráficas de crecimiento de la OMS parten de esta tradición y no describen simplemente el crecimiento de niños normales actuales. Más bien, aspiran a representar el crecimiento óptimo de los niños. Operativamente, esto se definió como el crecimiento de niños que vivían en condiciones desprovistas de cualquier impedimento ambiental sobre el crecimiento. En el caso de lactantes y niños que dan sus primeros pasos, representa además una alimentación conforme a las recomendaciones de la OMS. En consecuencia, las gráficas de la OMS se clasifican como 'patrones', en contraste con las 'referencias' que describen el crecimiento de todos los niños incluyendo los que no viven en condiciones favorables. Los patrones de la OMS también pretendían ser ampliamente representativos a nivel internacional.

Mientras se estaban desarrollando las nuevas gráficas de la OMS, se publicaron dos gráficas nuevas. Las gráficas EuroGrowth, resultantes de un estudio multicéntrico sobre crecimiento realizado en toda Europa [5], se publicaron en el año 2000. También en este mismo año, los Centros para el Control y la Prevención de Enfermedades (CDC) publicaron nuevas gráficas de crecimiento que se basaban en EE.UU. [6, 7] y eran el resultado de una revisión de las gráficas del NCHS emprendida con la intención de corregir las imperfecciones de estas últimas.

Aquí se presentan los nuevos patrones de crecimiento de la OMS, en comparación con las gráficas NCHS/OMS, a las que reemplazan, así como las gráficas Euro-Growth y CDC. Constatamos en qué aspectos difieren las gráficas de la OMS de las demás y también cómo las demás gráficas difieren entre sí. Aunque muchas de las diferencias son demasiado exiguas para ejercer algún impacto, algunas de ellas son bastante considerables y es probable que conlleven consecuencias funcionales.

\section{Los nuevos patrones de crecimiento de la OMS}

Se efectuó un estudio multicéntrico de referencia del crecimiento entre 1997 y 2003 con el objetivo de describir el crecimiento de niños que vivían en condiciones que no impedían el crecimiento. Debido a que la intención era crear un patrón representativo en el ámbito internacional, se recogieron datos en 6 lugares de 6 países (Brasil, Ghana, India, Noruega, Omán y EE.UU.). El estudio comprendía dos partes: un estudio longitudinal en el cual se seguía a los sujetos desde el nacimiento hasta los 2 años de edad y un estudio transversal en niños de edades comprendidas entre 1,5 y 5 años. Conviene destacar que, lo mismo que todas las demás gráficas, las gráficas de la OMS desde el nacimiento hasta los 2 años presentan la longitud del niño en decúbito y sólo a partir de 2 años en adelante representan la talla tomada de pie.

\section{Estudio longitudinal}

De los 1.737 sujetos incluidos en el estudio longitudinal, lo completaron 882 y sólo de éstos se utilizaron los datos. Se incluyeron lactantes nacidos a término, independientemente del peso al nacer, de manera que la muestra incluyese un $2,3 \%$ de lactantes con un peso al nacer de $<2.500 \mathrm{~g}$. Los sujetos fueron alimentados de conformidad con las recomendaciones de la OMS, lo que significa que fueron amamantados durante los 12 primeros meses de vida, introduciendo alimentos complementarios sólo al cabo de los 6 meses de edad. Sus madres no fumaban cigarrillos. La aplicación de criterios estrictos de elegibilidad en algunos países obligó a la exclusión de la mayoría de los sujetos en las márgenes de edad respectivas, mientras que en otros países los criterios de elegibilidad excluyeron únicamente una pequeña parte de los sujetos. Destaca también que los sujetos procedían, en general, de los estratos más privilegiados de las sociedades.

\section{Estudio transversal}

Los sujetos fueron reclutados en los mismos estratos demográficos que los sujetos del estudio longitudinal. Los 6.669 sujetos, de edades comprendidas entre 18 y 71 meses, fueron medidos, cada uno de ellos, en una sola ocasión. Dados los efectos adversos que causa el sobrepeso sobre la salud, de la muestra transversal se excluyeron los datos de los sujetos con un peso para la talla $>2$ DE. De este modo, se excluyeron datos del 1,4\% de los niños y el 1,1\% de las niñas.

\section{Otras gráficas de crecimiento}

Las Curvas de Crecimiento de 1977 del NCHS [1], desde el nacimiento hasta 3 años, se basaron en datos longitudinales recogidos por el Instituto Fels entre 1929 y 1975 [8]. Los sujetos eran predominantemente de raza blanca y vivían en una zona geográfica estrecha de Estados Unidos. Aunque la mayoría de los sujetos habían sido amamantados cuando eran lactantes, 
algunos lo fueron únicamente durante periodos cortos y una minoría considerable no recibió en absoluto leche materna. Las mediciones del peso y la longitud se efectuaron cada 3 meses durante el primer año de vida. Para las edades entre 2 y 18 años, el NCHS se basó en datos transversales representativos a nivel nacional procedentes de Encuestas Nacionales de Exámenes Sanitarios.

Las Gráficas del 2000 de los CDC [6, 7] representan una modificación de las gráficas del NCHS. Desde el nacimiento hasta los 3 años, los datos longitudinales originales fueron sustituidos por datos transversales de cinco encuestas, representativas a nivel nacional, efectuadas en EE.UU. entre 1963 y 1994. Debido a la precariedad de los datos de lactantes se utilizaron datos adicionales de longitud (pero no de peso) de clínicas de salud auspiciadas por el gobierno (mujeres, lactantes y niños). En conjunto, se dispuso de datos del peso únicamente para menos de 300 sujetos/mes (niños y niñas) entre 3 y 12 meses de edad (ausencia de datos del peso al mes y a los 2 meses de edad). Se dispuso de datos de longitud de una considerable cantidad de sujetos de 1 a 5 meses de edad, si bien de menos de 300/mes entre 6 y 12 meses de edad. Debido al número limitado de sujetos, la confianza en la exactitud de los percentiles externos es baja. No se dispone de información sobre la nutrición de los lactantes. Se excluyeron los datos del peso de los niños mayores de 6 años, obtenidos entre 1988 y 1994, debido a la elevada prevalencia en esta cohorte de niños con pesos 'insanos' (es decir, elevados).

Gráficas Euro-Growth 2000 [5]. Los datos de las gráficas Euro-Growth se recogieron en 22 lugares de 11 países europeos. Se siguió longitudinalmente a los sujetos nacidos entre 1990 y 1993 desde el nacimiento hasta los 5 años. De 2.245 sujetos incluidos, 1.746 completaron el estudio hasta los 12 meses de edad, 1.205 hasta los 24 meses y 1.071 hasta los 3 años. Los sujetos nacieron después de las 37 o más semanas de gestación con un peso al nacer mayor de $2.500 \mathrm{~g}$. Los sujetos alimentados de acuerdo a las recomendaciones de la OMS representaban una minoría. Tales recomendaciones consisten en lactancia materna durante un año, con alimentos complementarios sólo después de los 6 meses de edad.

\section{Evaluación funcional}

Se evaluó el impacto funcional de las diferencias entre las gráficas con la ayuda de muestras hipotéticas. Estas muestras presentaban un peso y una longitud/talla distribuidos normalmente y se centraron en el percentil 50 de las gráficas del NCHS.

\section{Los patrones de la OMS en comparación}

\section{Primer año de vida}

En la figura 1 se ilustran los percentiles 5, 50 y 95 del peso para la edad de niños durante el primer año de vida, correspondientes al nuevo patrón de crecimiento de la OMS en com-

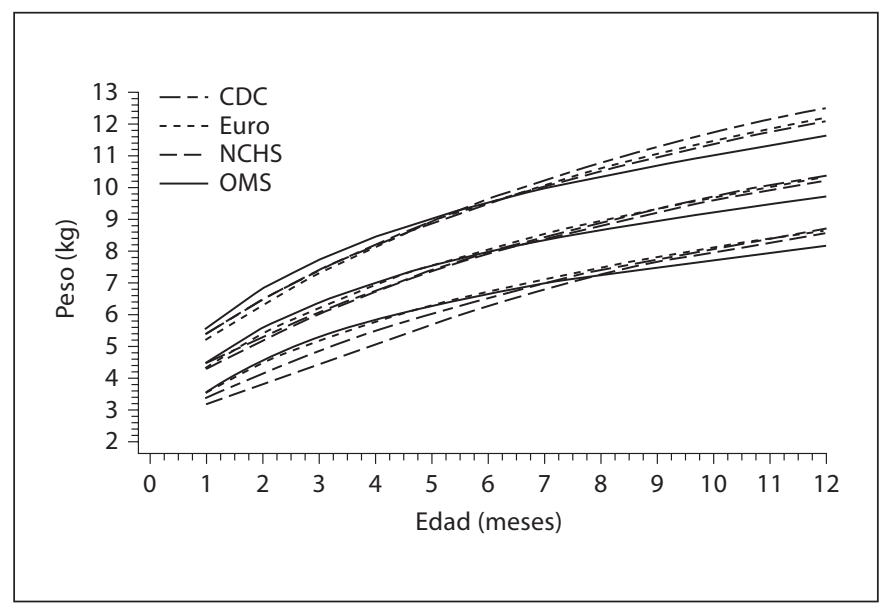

Fig. 1. Peso para la edad de lactantes varones de 1 a 12 meses de edad. Se ilustran los percentiles 5, 50 y 95 para las gráficas de la OMS en comparación con las gráficas de Euro, NCHS y CDC.

paración con las otras tres gráficas (las relaciones entre las gráficas son en cada caso similares para las niñas, por lo que las gráficas para las niñas no se muestran por separado). Es evidente que durante los 6 primeros meses el peso de la OMS tiende a ser mayor que el peso de las demás gráficas en todos los percentiles. Durante los segundos 6 meses de vida ocurre a la inversa, siendo el peso de la OMS progresivamente menor que el peso de las otras gráficas. La magnitud de las diferencias no es la misma para todos los percentiles. Durante los 6 primeros meses las diferencias son especialmente considerables para el percentil 5, mientras que durante el segundo semestre las diferencias son más bien homogéneas. Para facilitar la comparación, la figura 2 ilustra el percentil 50 de las demás gráficas, expresado en unidades DE (puntaje z) de los patrones de la OMS. Durante los 6 primeros meses, las diferencias alcanzan 0,3 a 0,6 unidades DE. Después de 6 meses, las diferencias alcanzan alrededor de 0,5 unidades $\mathrm{DE}$ a los 12 meses, si bien en la dirección opuesta.

Con respecto a la longitud (fig. 3), las diferencias entre los patrones de la OMS y otras gráficas son menos espectaculares que para el peso. La dispersión entre los percentiles externos tiende a ser más estrecha para los patrones de la OMS que para las demás gráficas. Tal como se ilustra en la figura 4 , las diferencias en el percentil 50 tienden a ser inferiores a 0,3 unidades DE. Contrariamente al peso de los CDC, la longitud de los CDC difiere notablemente de la longitud del NCHS y la longitud de Euro-Growth; no se dispone de ninguna explicación de esta discrepancia.

Para obtener cierta perspectiva sobre las fuentes de las diferencias entre las gráficas se utilizaron datos del crecimiento publicados $[2,9,10]$ y no publicados de lactantes alimentados con leche materna o alimentados con leches para lactantes. La 


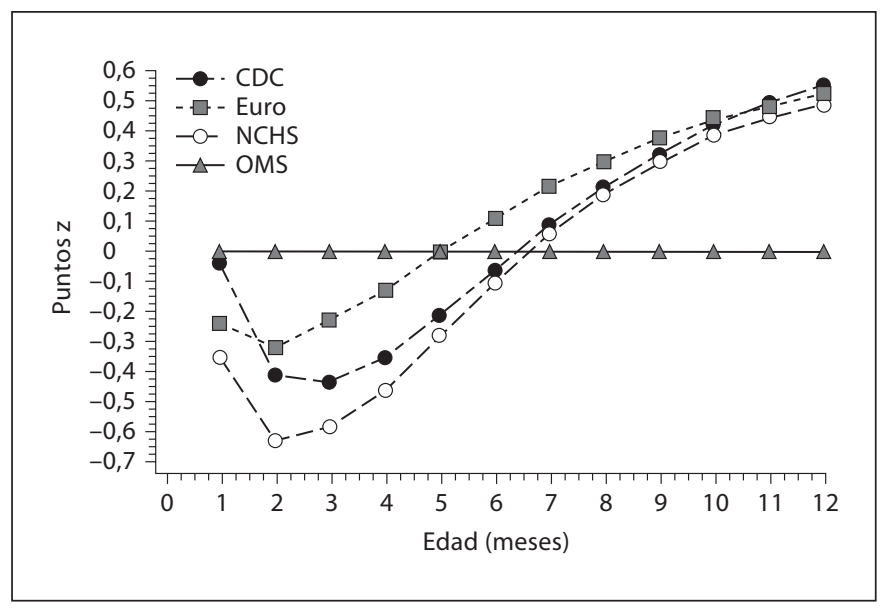

Fig. 2. Percentil 50 del peso para la edad (lactantes masculinos y femeninos) de las gráficas Euro, CDC y NCHS, expresado como puntos z de las gráficas de la OMS.

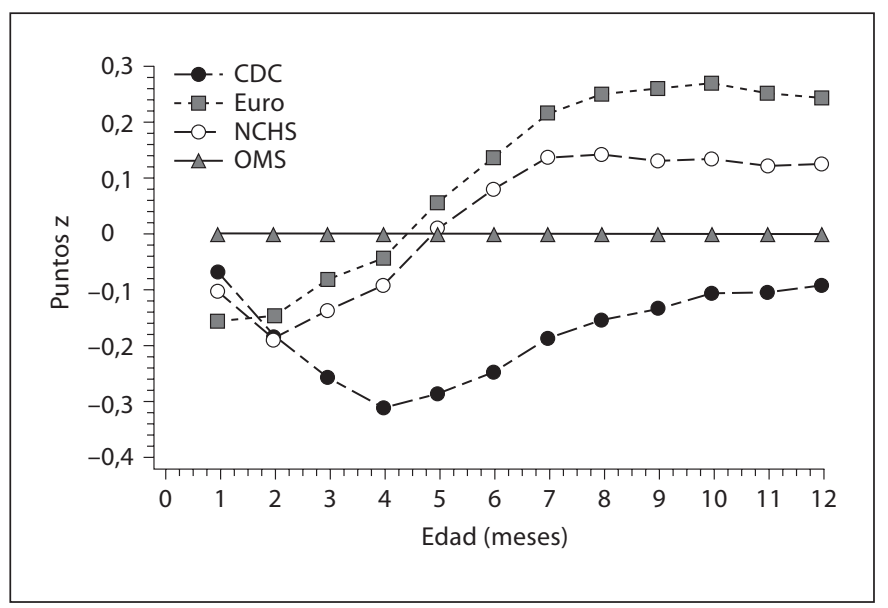

Fig. 4. Percentil 50 de la longitud para la edad (lactantes masculinos y femeninos) de las gráficas Euro, CDC y NCHS, expresado como puntos z de la OMS.

comparación con estos datos (no expuestos) lleva a la conclusión de que durante los 6 primeros meses OMS y Euro-Growth representan más fielmente el crecimiento de los lactantes que NCHS y CDC. Las diferencias en el peso entre los bebés alimentados con leche materna y alimentados con leches para lactantes son mucho menores que las diferencias entre la OMS por una parte y las gráficas de NCHS y CDC por otra. Esto lleva a la conclusión adicional de que las imperfecciones de las gráficas del NCHS, y también las gráficas de los CDC, en los 6 primeros meses de vida no constituyen un reflejo de diferencias en las

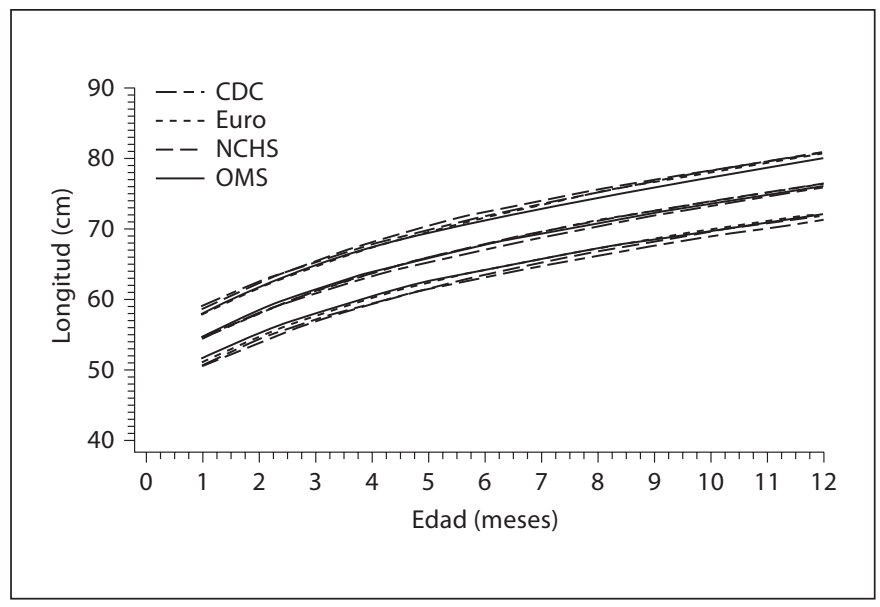

Fig. 3. Longitud para la edad de lactantes varones de 1 a 12 meses de edad. Se ilustran los percentiles 5, 50 y 95 para las gráficas de la OMS en comparación con las gráficas de Euro, NCHS y CDC.

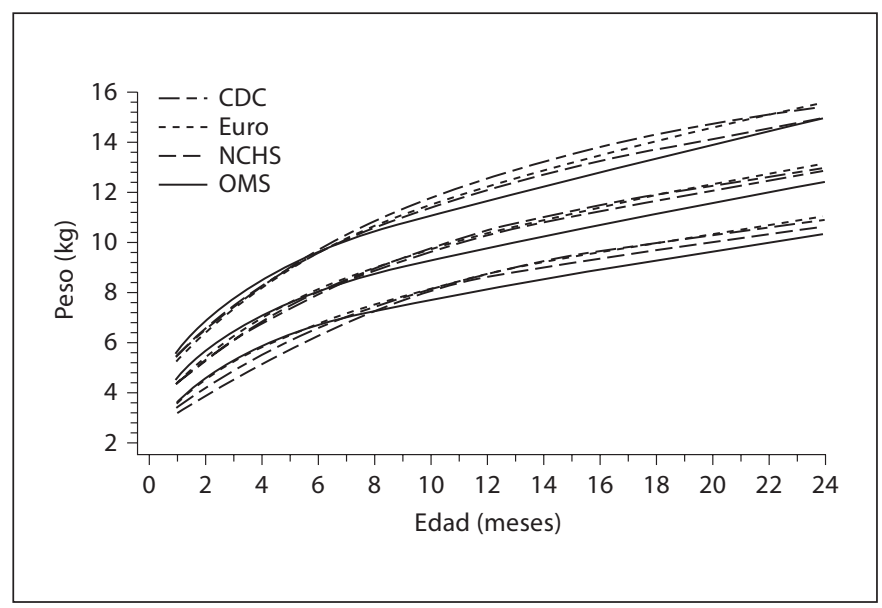

Fig. 5. Peso para la edad de niños varones de 12 a 24 meses de edad. Se ilustran los percentiles 5, 50 y 95 para las gráficas de la OMS en comparación con las gráficas de Euro, NCHS y CDC.

modalidades alimentarias (es decir, amamantamiento frente a alimentación con leches para lactantes), sino que más bien sus orígenes son ajenos. Se supone que se deben, por ejemplo, a la precariedad de las mediciones $y$, probablemente, los métodos de ajuste y aplanamiento de las curvas. Estas comparaciones también llevaron a la conclusión de que las gráficas de los CDC no representan una mejora sustancial capaz de superar la insuficiencia de sus predecesoras, las gráficas del NCHS. Ni las gráficas del NCHS ni las de los CDC representan muy correctamente el crecimiento de lactantes en los 6 primeros meses. 


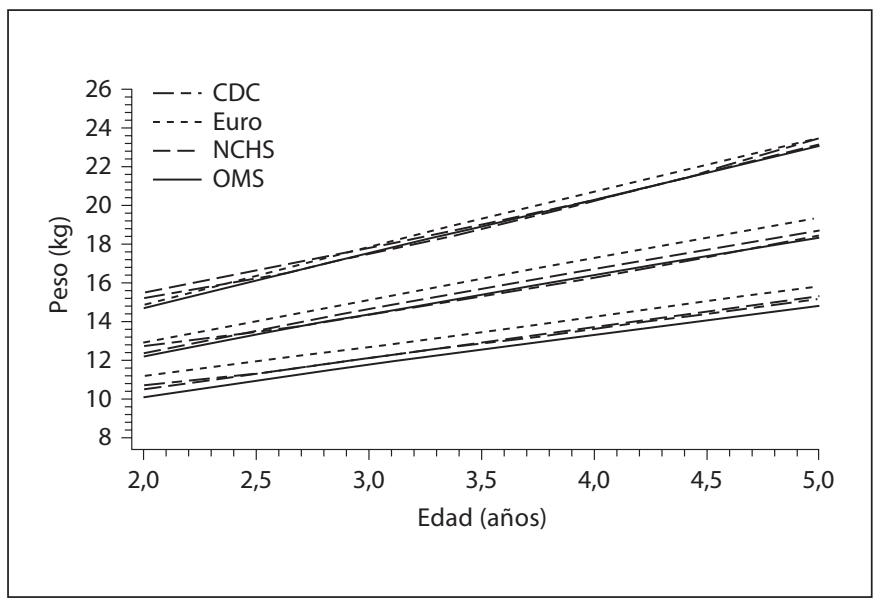

Fig. 6. Peso para la edad de niños varones de 2 a 5 años de edad. Se ilustran los percentiles 5, 50 y 95 para las gráficas de la OMS en comparación con las gráficas de Euro, NCHS y CDC.

Segundo año de vida

Las tendencias y las diferencias que aparecen durante los segundos 6 meses del primer año continúan durante el segundo año de vida. Tal como se ilustra en la figura 5, todos los percentiles de peso de las gráficas de la OMS son menores que los de las demás gráficas de crecimiento. Los percentiles de longitud (no expuestos) continúan análogamente sobre trayectorias establecidas entre 6 y 12 meses, al respecto de lo cual las gráficas de la OMS siguen mostrando una dispersión más estrecha entre los percentiles externos que otras gráficas.

\section{De dos a cinco años}

Entre 2 y 5 años hay diferencias notables entre las gráficas en cuanto al peso (fig. 6) y la talla (fig. 7). El peso de EuroGrowth (en contraste con la longitud) tiende a ser mayor que el peso de otras gráficas en una medida considerable $(0,4$ a 0,6 unidades DE), para lo cual no se dispone de una explicación convincente. Los percentiles de peso de la OMS tienden a ocupar el extremo inferior del espectro. Esto se explica, por lo menos para los percentiles superiores, por la exclusión de datos de algunos de los niños con más peso. La pauta peculiar del peso de los CDC entre 2 y 3 años resulta de la vinculación entre las gráficas para lactantes y las gráficas para niños.

\section{Evaluación funcional}

En la figura 8 se comparan los porcentajes de niños normales de 1 a 2 años de edad, identificados por tener un peso inferior al ideal (peso < percentil 10). La proporción de niños normales identificados como poseedores de un peso inferior al ideal es notablemente menor con el uso de las gráficas de la OMS que con las demás gráficas, si bien las diferencias entre las otras gráficas son también considerables. Utilizando las

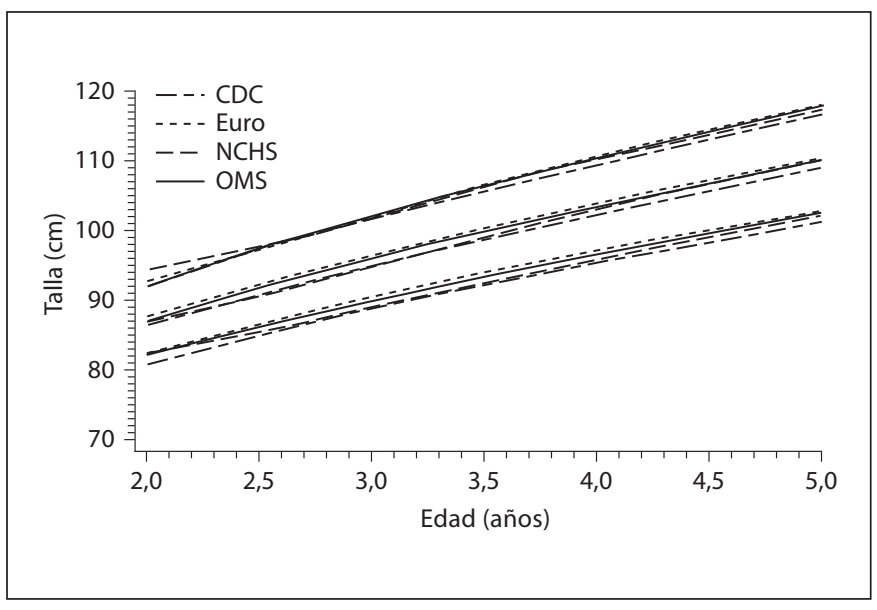

Fig. 7. Talla para la edad de niños varones de 2 a 5 años de edad. Se ilustran los percentiles 5, 50 y 95 para las gráficas de la OMS en comparación con las gráficas de Euro, NCHS y CDC.

gráficas de la OMS, alrededor del 3\% de los niños se identifican con un peso inferior al ideal, mientras que utilizando las gráficas del NCHS se identifican alrededor del $8 \%$ en esa categoría; con las curvas CDC y Euro, la identificación se eleva hasta el 13-14\% aproximadamente. Es de suponer que el empleo de las nuevas gráficas de la OMS podría ser la causa de una demora en la identificación del retraso en el crecimiento que aparece en lactantes o niños que dan sus primeros pasos alimentados con leches para lactantes.

Otro ejemplo se ilustra en la figura 9. En niños normales de 2 a 5 años de edad, la proporción identificada como afectados de sobrepeso (IMC > percentil 90) es del 16\% aproximadamente con el uso de las gráficas de la OMS, en contraste con sólo el $11 \%$ con las gráficas de los CDC y alrededor del 7\% con las gráficas Euro-Growth.

Estos ejemplos ilustran las considerables diferencias en las proporciones de niños identificados cuando se utilizan las diversas gráficas para propósitos de detección sistemática. Aunque existen diferencias entre todas las gráficas, para algunas de las variables más importantes (fig. 8 y 9) las gráficas de la OMS se sitúan en el extremo del espectro. Este es en gran medida el efecto pretendido por el enfoque prescriptivo utilizado por la OMS.

\section{Comentario}

Las nuevas gráficas de crecimiento de la OMS, que fueron creadas utilizando un enfoque prescriptivo, aspiran a describir el crecimiento de niños como si su curso discurriera de un modo óptimo y no estuviera dificultado por factores externos. Las gráficas de crecimiento de la OMS difieren de las gráficas 


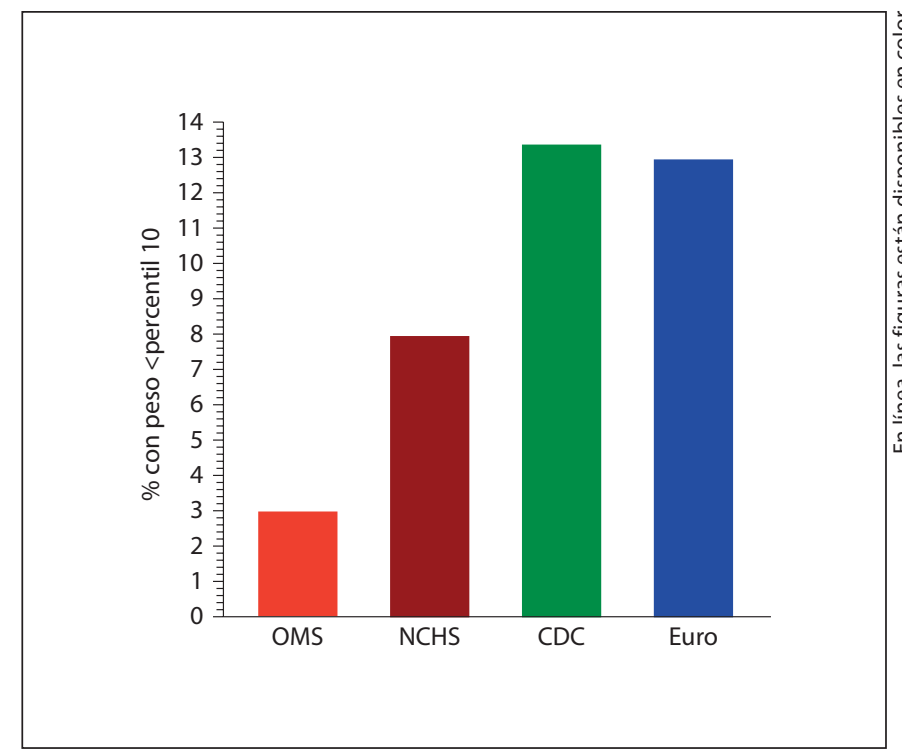

Fig. 8. Porcentaje de niños normales de 1 a 2 años de edad, identificados con un peso inferior al ideal utilizando las gráficas de crecimiento de OMS, NCHS, CDC o Euro.

de crecimiento existentes en aspectos importantes. Aunque hay numerosas diferencias entre las gráficas de crecimiento existentes, se distinguen por seguir una pauta diferente. Esto es en gran medida el resultado del enfoque prescriptivo. Las diferencias distan mucho de ser uniformes, dado que cambian de dirección y varían en magnitud con la edad. Por ejemplo, en los 6 primeros meses, el peso de la OMS es mayor que el peso en cualquier otra gráfica, mientras que en los segundos 6 meses, el peso de la OMS es menor que el peso en cualquiera de las demás gráficas. Las diferencias en el peso durante los 6 primeros meses no pueden explicarse por completo por las diferencias en las modalidades alimentarias y son en parte la consecuencia de otros factores, predominantemente de naturaleza técnica.

Para el empleo de las gráficas en el tratamiento de niños individuales (uso clínico), es probable que las diferencias entre las gráficas conlleven una consecuencia de carácter menor. Después de todo, el médico suele evaluar el crecimiento basándose en mediciones múltiples y tiende a juzgar el crecimiento por la medida en la que sigue su curso en paralelo a los percentiles. Por lo tanto, para el uso clínico, la posición de los percentiles tiene menos importancia que la dirección de los mismos. Por otra parte, en el uso epidemiológico de las gráficas de crecimiento, donde el objetivo consiste en determinar la prevalencia del crecimiento aberrante, la posición de los percentiles externos concita un interés máximo. En este caso, las diferencias entre las gráficas de crecimiento pueden ejercer un impacto considerable. Como ilustran los ejemplos

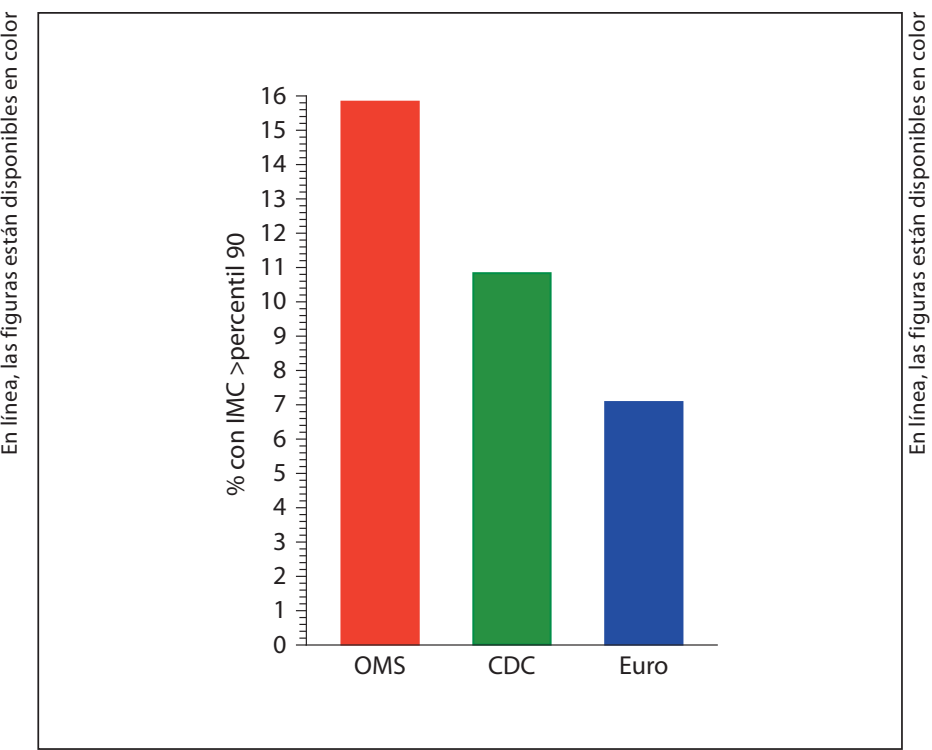

Fig. 9. Porcentaje de niños normales de 2 a 5 años de edad, identificados con sobrepeso utilizando las gráficas de crecimiento de OMS, CDC o Euro.

de las figuras 8 y 9, la proporción de sujetos identificados por las diversas gráficas varía considerablemente. En el caso de la gráfica de la OMS, este efecto es intencional. La gráfica de la OMS desea, por ejemplo, identificar más niños con sobrepeso que otras gráficas, presumiblemente porque de este modo el problema del sobrepeso en la infancia recibiría más atención. En consecuencia, el ajuste de los valores límite tradicionales parecería anular la intención de las gráficas de la OMS.

\section{Bibliografía}

Hamill PV, Drizd TA, Johnson CL, Reed RB, Roche AF: NCHS growth curves for children birth-18 years. United States. Vital Health Stat 11 1977; 165:i-iv, 1-74.

2 Dewey KG, Peerson JM, Brown KH, Krebs NF, Michaelsen KF, Persson LA, Salmenpera L, Whitehead RG, Yeung DL; WHO Working Group on Infant Growth: Growth of breast-fed infants deviates from current reference data: a pooled analysis of US, Canadian and European data sets. Pediatrics 1995;96:495-503.

3 de Onis M, Habicht JP: Anthropometric reference data for international use: recommendations from a World Health Organization Expert Committee. Am J Clin Nutr 1996;64:650-658. 
4 WHO Multicentre Growth Reference Study Group: WHO child growth standards based on length/height, weight and age. Acta Paediatr Suppl 2006;450:76-85.

5 Haschke F, van t'Hoff MA (eds): EuroGrowth. J Pediatr Gastroenterol Nutr 2000; 31(suppl 1):S1-S86.

6 Kuczmarski RJ, Ogden CL, Grummer-Strawn LM, Flegal KM, Guo SS, Wei R, Mei Z, Roche AF, Johnson CL: CDC growth charts: United States. Adv Data 2000;314:1-27.
7 Ogden CL, Kuczmarski RJ, Flegal KM, Mei Z, Guo S, Wei R, Grummer-Strawn LM, Curtin LR, Roche AF, Johnson CL: Centers for Disease Control and Prevention 2000 growth charts for the United Sates: improvements to the 1977 National Center for Health Statistics version. Pediatrics 2002;109:45-60.

8 Roche AF: Growth, Maturation and Body Composition: The Fels Longitudinal Study 1929-1991. Cambridge, Cambridge University Press, 1992.
9 Nelson SE, Rogers RR, Ziegler EE, Fomon SJ: Gain in weight and length during early infancy. Early Hum Dev 1989;19:223-239.

10 Haschke F, van t'Hoff MA; Euro-Growth Study Group: Euro-Growth references for breast-fed boys and girls: influence of breastfeeding and solids on growth until 36 months of age. J Pediatr Gastroenterol Nutr 2000; 31(suppl 1):S60-S71. 